Incidence and persistence of depression in HIV in Uganda

\title{
Incidence and persistence of major depressive disorder among people living with HIV in Uganda
}

Eugene Kinyanda ${ }^{1,2,3}$, Helen A. Weiss ${ }^{3}$, Jonathan Levin ${ }^{4}$, Noeline Nakasujja ${ }^{2}$, Harriet Birabwa ${ }^{5}$, Juliet Nakku , Richard Mpango $^{1}$, Heiner Grosskurth ${ }^{3}$, Soraya Seedat ${ }^{6}$, Ricardo Araya $^{3}$, Vikram Patel $^{3,7}$

$\underline{\text { Institutional Affiliation }}$

${ }^{1}$ Mental Health Project, MRC/UVRI Uganda Research Unit on AIDS/ MRC-DFID African Leadership Award, Entebbe, Uganda

${ }^{2}$ Department of Psychiatry, Makerere College of Health Sciences, Kampala, Uganda

${ }^{3}$ London School of Hygiene and Tropical Medicine, London, United Kingdom

${ }^{4}$ School of Public Health, University of the Witwatersrand, Johannesburg, South Africa.

${ }^{5}$ Butabika National Psychiatric Referral Hospital, Kampala, Uganda

${ }^{6}$ Department of Psychiatry, Stellenbosch University, Cape Town, South Africa

${ }^{7}$ Senior Wellcome Trust Fellowship, London, United Kingdom

\section{Correspondent author}

Eugene Kinyanda, Mental Health Project, MRC/UVRI Uganda Research Unit on AIDS, Entebbe, Uganda. P.O. Box 49 Entebbe, Uganda. Telephone: +256417704159; Email: Eugene.Kinyanda@mrcuganda.org . 
Incidence and persistence of depression in HIV in Uganda

\begin{abstract}
Data on the course of major depressive disorder (MDD) among people living with HIV (PLWH) are needed to inform refinement of screening and interventions for MDD. This paper describes the incidence and persistence rate of MDD in PLWH in Uganda. 1099 ART-naïve PLWH attending HIV clinics in Uganda were followed up for 12 months. MDD was assessed using the DSM IV based Mini-International Neuropsychiatric Interview with a prevalence for MDD at baseline of $14.0 \%$ (95\% CI1 $1.7 \%-16.3 \%$ ) reported. Multivariable logistic regression was used to determine predictors of incident and persistent MDD. Cumulative incidence of MDD was 6.1 per 100 person-years (95\%CI 4.6-7.8) with significant independent predictors of study site, marital status, higher baseline depression score and increased stress. Persistence of MDD was 24.6\% (95\%CI 17.9\%-32.5\%) with independent significant predictors of study site, religion, higher baseline depression score, and increased weight. Risks of incident and persistent MDD observed in this study were high. Potentially modifiable factors of elevated baseline depressive scores and stress (only for incident MDD) were important predictors of incident and persistent MDD.
\end{abstract}

Key Words: HIV/AIDS, Major depressive disorder, incidence, persistence, predictors 
Incidence and persistence of depression in HIV in Uganda

\section{Introduction}

Major depressive disorder (MDD) is the most common neuropsychiatric complication among people living with HIV (PLWH) with prevalence ranging from $8 \%$ to $60 \%$ [1-7]. Negative impacts of MDD among PLWH include poorer quality of life, faster HIV disease progression, higher mortality, poor adherence to medication regimens, increased risky sexual behaviour, and perpetration of intimate partner violence [2,6-9]. Most studies of MDD among PLWH have been cross-sectional, and provided limited evidence on the course and aetiology of MDD due to the episodic nature of MDD $[4,10]$ and the bi-directional relationship between MDD and HIV/AIDS [6]. Elucidating the course of MDD in HIV/AIDS and it's predictors is important for refining screening procedures for MDD in HIV/AIDS and for the design and evaluation (including clinical trials) of intervention against MDD in HIV/AIDS.

Globally, a number of longitudinal studies on MDD among PLWH have been undertaken in both high-income countries [5,11-15] and in low and-middle income countries [4, 7, 9, 16-18]. However to date, only two such studies have been undertaken in sub-Saharan Africa [4,7]. Previous studies that have looked at the incidence of MDD in HIV/AIDS have reported rates of between 1.89 to 2.2 to per 100 person-years [17-18]. Most of the prospective studies (with none from a developing country context) that have included a non-HIV positive control group have reported no difference or only a slightly increased incidence of MDD associated with HIV [16-18]. From high income settings, predictors of incident MDD in PLWH included advanced HIV clinical stage, more frequent clinic visits, multiple psychiatric comorbidity, past history of MDD and social conflict, [3,12,13]. From developing country settings, predictors of incident MDD in PLWH included advanced HIV clinical stage, more frequent clinic visits, disability in work/social/family functioning, greater number of negative life events, decline in CD4 counts, HIV diagnosis made less than one year ago, being in HIV care for less than one year, having comorbid anxiety and female gender [4,16-18].

Similarly, there have been few studies on persistence of MDD among PLWH. In one of the pioneering studies in this area by Lyketsos and colleagues (1996) who followed up HIV positive 
Incidence and persistence of depression in HIV in Uganda

patients not on antiretroviral therapy (ART) over a 10 year period reported a dramatic sustained rise in depressive symptoms beginning 18 months before the development of AIDS and peaking at diagnosis of clinical AIDS [11]. In a more recent South African study where 29 participants with HIV and MDD were followed up over 6-months, the risk of persistence for MDD was 44.8\% [4]. Predictors of persistence of MDD in HIV/AIDS include elevated depressive symptoms in early stage disease, selfreport of AIDS-related symptoms (fatigue and rash), concurrent unemployment, cigarette smoking, limited social supports and high baseline HIV symptom count [11,14].

This paper will describe the epidemiology of incidence and persistence of MDD among PLWH followed for 12 months in Uganda.

\section{Methods}

\section{Study design and Site}

This is a prospective cohort study where assessments were undertaken at baseline, 6-months and 12months. This study was undertaken at two specialised HIV clinics run by the AIDS Support Organisation (TASO) at Entebbe (semi-urban) and Masaka (predominantly rural) [19]. The MRC/UVRI Uganda Research Unit (the host research institution) has an established research collaboration with these two study clinics. The TASO Entebbe clinic has approximately 7,000 active clients of whom about 3,000 are ART-naïve while the Masaka TASO clinic has approximately 6,000 clients of whom about 2,500 are ART-naïve.

\section{Sampling Procedure}

This study aimed to enrol 1100 ART naïve HIV positive adults from the TASO HIV clinics in Masaka and Entebbe (rural and semi-urban). We estimated that the sample size of 1100 respondents would allow us to estimate the actual incidence of MDD to a precision of about \pm 1.4 per 100 PYO based on the following assumptions: the incident MDD cases will follow a Poisson distribution, baseline prevalence of MDD will be $11 \%$; and incident rate of MDD will be 5 cases per 100 PYO. To obtain this sample, a sub-register of all active clients who were not on ART was created. From this register a 
Incidence and persistence of depression in HIV in Uganda

random sample of 550 ART naïve patients was recruited from each clinic using a table of random numbers.

The inclusion criteria for this study were: i) A person living with HIV/AIDS who was ART naïve and registered with the outpatient clinic at either TASO Entebbe and TASO Masaka clinics; ii) Aged at least 18 years old at enrolment; iii) Conversant in Luganda, the language into which the research protocols were translated (the majority of respondents were not conversant with English the language in which the standardised assessment tools were developed). Exclusion criteria were being too sick or unable to understand the study instruments, and having defaulted on the most recent previous scheduled clinic visit. Eligible participants were asked for written informed consent after explanation of the study objectives and procedures.

\section{Data collection tools}

The data collection tools consisted of structured and standardised locally translated psychosocial assessment tools most of which have previously been used among PLWH in the Ugandan setting by this study group [1]. The psychosocial assessment tools being employed for the first time in the Ugandan HIV setting were taken through a process of locally adaptation. This involved a process of forward and back translation by two teams of mental health professionals conversant with both English and the local language of translation (Luganda) working independent of each other. At a consensus meeting where both these teams were represented the final back translated English version was then compared to the initial English version. On those items where there was wide variation in the two versions, a consensus position was arrived at through discussion. The internal consistency of each of all the tools being used for the first time was determined by assessing their Cronbach's alpha and only those tools which had Cronbach's alpha of at least 0.7 were included in subsequent analysis for this study.

The data collection tools for this study were compiled together into the following groups: sociodemographic factors (Group 1), HIV associated clinical factors (Group 2A), vulnerability/protective factors (Group 2B), stressors (Group 2C) and the outcome measures of MDD as specified by the 
Incidence and persistence of depression in HIV in Uganda

conceptual framework for this study which is based on the stress-vulnerability model for depression (Figure 1) [20].

\section{Insert Figure 1}

A diagnoses of MDD was made using the DSM IV based Mini-International Neuropsychiatric Interview [31] while severity of depressive symptoms was assessed using the Centre for Epidemiological Studies-Depression questionnaire (CES-D) [32], the rest of the data collection tools are listed in Table I below.

\section{Insert Table I}

\section{Statistical analysis}

There were two dependent variables: i) incident major depressive disorder(MDD) assessed at 6months and 12- months amongst those without MDD at baseline; and ii) persistent MDD over the 612 month follow-up among those with MDD at baseline (i.e. MDD detected at either 6 or 12 month follow-up). Logistic regression was used to estimate odds ratios and 95\%CIs. A conceptual framework (see Figure 1) based on the stress-vulnerability models of depression [20] was developed $a$ priori to guide the multivariable analysis [33]. Firstly, socio-demographic factors were included if there was at least weak evidence of a univariable association $(p<0.15)$. Factors were retained if they remained with $\mathrm{P}<0.15$ after adjustment for other socio-demographic factors. The liberal $\mathrm{P}$-value cutoff was used in order to ensure that all variables that could have a possible confounding effect on the ultimate risk factors were included [34].

According to the conceptual framework, the socio-demographic variables (Group 1 variables) may act on MDD through three groups of proximal factors, a set of HIV associated clinical variables (Group 2A), a set of vulnerability/protective variables (Group 2B), and a set of stressor variables (Group 2C). In turn, the HIV associated clinical variables may act through Groups 2B and 2C as well 
Incidence and persistence of depression in HIV in Uganda

as independently on MDD. Thus three second stage models were fitted, involving the selection of Group 1, and Group 2A, 2B or 2C variables respectively. The HIV associated clinical factors (Group 2A), vulnerability/protective factors (Group 2B), and stressor factors (Group 2C) associated with the outcome $(\mathrm{P}<0.15)$ after adjusting for the Group 1 variables and baseline CES-D score were selected into an initial multivariable model. The final multivariable model included variables that were independently associated with the outcome $(\mathrm{P}<0.10)$, using a backward elimination method. Part of the effect of the socio-demographic variables will be mediated through the vulnerability/protective variables, HIV associated clinical variables or through the stress variables, so the selected sociodemographic variables were not removed from the final model to capture both their direct and indirect effects [33]. For all continuous explanatory variables, we explored fractional polynomials of degrees 1 and 2 to investigate for potential nonlinearity [34].

\section{Ethical Considerations}

The study obtained ethical approval from the Uganda Virus Research Institute's Science and Ethics Committee, the London School of Hygiene and Tropical Medicine Ethical Committee and the Uganda National Council of Science and Technology. Study participants were invited to consent after being provided with adequate information about the study. Respondents found to have significant psychiatric problems were referred to the psychiatric departments at Entebbe district hospital (at the semi-urban site) and Masaka regional referral hospital (rural site) for further assessment and management.

\section{Results}

Of the 1099 participants assessed at baseline, 68 (6.2\%) were lost to follow-up by 12 months, of whom 18 were confirmed to have died during the course of this study, the majority due to non HIV related causes. None of the factors of study site, gender, marital status and baseline MDD were associated with loss to follow-up. 
Incidence and persistence of depression in HIV in Uganda

Amongst the 944 participants without MDD at baseline, 920 had follow-up data, and there were 56 incident cases over the 12 month follow-up period (6.1\%; Table II). Amongst the 155 participants with MDD at baseline, 146 had follow-up data, including 36 persistent cases (24.7\%), including 11 recurrent cases who were positive-negative-positive (Table II).

\section{Insert Table II}

\section{Characteristics of study population}

The majority $(\mathrm{n}=847,77.1 \%)$ of the study respondents were female. The mean age was 35.1 years (standard deviation $(\mathrm{SD})=9.3)$, and the majority were in early HIV stage disease $(48.5 \%$ Stage 1 ;

45.5\% Stage 2; 6.0\% Stage 3) with the majority having CD4 counts above 350 cells/ul $(<201$ cells/ul $=9.4 \% ; 201-350$ cells $/ \mathrm{ul}=15.3 \% ; 351-500$ cells $/ \mathrm{ul}=30.5 \% ;$ and $>500=44.9 \%)$.

\section{Incidence of MDD and risk of persistence of MDD}

The cumulative incidence of MDD over 12 months in this study was 6.1 per 100 person- years (95\%CI 4.6-7.8). The risk of persistent of MDD over 12 months was $24.6 \%$ (95\%CI 17.9\%-32.5\%).

\section{Univariate predictors of incident MDD}

\section{Insert Table III}

Table III shows factors associated with incident MDD. Among the socio-demographic factors, the only factor associated with incident MDD was study site (OR for Masaka vs Entebbe $=4.35,95 \% \mathrm{CI}$ 2.27-8.36). After adjusting for study site, the following factors were associated with incident MDD: higher disability scores $(\mathrm{OR}=1.39,95 \% \mathrm{CI} 1.08-1.80)$; higher negative life events scores $(\mathrm{OR}=1.65$, 
Incidence and persistence of depression in HIV in Uganda

95\%CI 1.25-2.17); andhigher stress scores (OR=1.64, 95\%CI 1.26-2.14); approaching significance were lower social support scores ( $\mathrm{OR}=0.79,95 \% \mathrm{CI} 0.61-1.02)$ and baseline depressive symptom scores $(\mathrm{OR}=1.11,95 \% \mathrm{CI} 0.98-1.27)$.

\section{Univariate predictors of persistent MDD}

\section{Insert Table IV}

Table IV shows univariate predictor factors that were associated with persistent MDD. Among the sociodemographic factors, study site (Masaka-rural site) and religion were marginally associated with persistent MDD. Baseline depressive symptom scores were associated with persistent MDD after adjusting for study site and religion. After adjusting for study site, religion and baseline depressive symptom score (CES-D), the following variables were marginally associated with persistent MDD: lower CD count, a heavier weight, family history of psychiatric disorder $(\mathrm{OR}=2.25$, 95\%CI 0.98-5.12); higher negative life events score ( $\mathrm{OR}=1.37,95 \% \mathrm{CI} 0.92-2.04)$ and higher stress scores (OR=1.34, 95\%CI: 0.90-1.98).

\section{Multivariate predictors of both incident MDD and persistent MDD}

\section{Insert Table V}

Table V shows the independent factors associated with incident MDD and persistent MDD respectively. Living in the rural site (Masaka) was independently associated with both incident and persistent MDD, as was a higher baseline CES-D score. An increased stress score was associated with incident $\mathrm{MDD}(\mathrm{OR}=1.56,95 \% \mathrm{CI} 1.18-2.05)$ and a heavier weight was associated with persistent $\operatorname{MDD}(\mathrm{OR}=1.47,95 \% \mathrm{CI} 0.93-2.33)$.

\section{Discussion}

This is one of few papers that have investigated the incidence and persistence of major depressive disorder (MDD) and their predictors in HIV/AIDS in the sub-Saharan African setting. The loss to 
Incidence and persistence of depression in HIV in Uganda

follow-up in this study was $6.2 \%$ (equivalent to 6.2 per 100 person-years) a figure much better than that reported by Olley and colleagues (2006) [4] in Cape Town, South Africa (56\%) but lower than that reported by Elenga and colleagues (2013) [17] in Guadeloupe in central America (1.6 to 2.7 per 100 person-years). In this study the factors of study site, gender, marital status and baseline MDD were not associated with loss to follow-up. In Guadeloupe in central America, Elenga and colleagues (2013) [17] have suggested that addiction may have been a factor in loss to follow-up in their study, a factor that was not investigated in this study.

The incidence of MDD in HIV/AIDS in this study was 6.1 per 100 person-years) a figure higher than that reported in Guadeloupe in Central America (2.2 per 100 person years) [17] and French Guyana in South America (1.89 per 100 person years) [18], these two countries having generalised HIV epidemics similar to the HIV epidemic in sub-Saharan Africa. Differences in the incidence of MDD between this study and those two previous studies could be due to a number of factors including: differences in the assessment of MDD (in this study we used a DSM IV based structured interviewthe M.I.N.I. [31] while in the two previous studies they used non-structured clinical interviews); differences in the risk for MDD associated with the parent HIV risk group (thought to be minimal as the HIV epidemic in all three studies was generalised); differences in the ecological risk factors for MDD and differences in the HIV clinical stage profiles of the three studies.

Predictors of incident MDD observed in this study included: study site (higher in the rural than urban site), greater disability, higher negative life events, higher stress and approaching significance were lower social support and baseline depressive scores. The independent predictors of incident MDD in this study were study site, elevated baseline depressive symptom scores and higher stress. Just as in this study, Olley and colleagues (2006) [4] in South Africa reported that disability in work, social and family functioning and a greater number of negative life events were predictors of incident MDD. Lyketsos and colleagues (1996) [11] in the USA reported that elevated depressive symptom scores 
Incidence and persistence of depression in HIV in Uganda

were a predictor of higher rates of depression as AIDS developed. More recently, Atkinson and colleagues (2008) [5] reported that a past history of MDD was associated with a four-fold increased risk for developing incident MDD. Similar to this study, Lackner and colleagues (1993) [35] in a follow up study of male homosexuals at risk of HIV observed that social support only marginally accounted for future MDD episodes. A previous cross sectional study by this research group in semiurban Entebbe (one of the study locations for this study) reported that increasing stress scores among HIV/AIDS patients was independently associated with prevalent MDD [1].

The association between study site and incident MDD observed in this study could not readily be explained. A possible explanation of this finding could be that study site represents ecological risk factors for MDD with this risk being greater in the rural area than in the urban area. Kinyanda and colleagues (2011) [36] in a community study that investigated district level indices to explain variation of district rates of MDD reported that only district literacy rates showed a reciprocal relationship with district rates of MDD. Comparing the two study sites on a few of the socioeconomic indices collected in this study reveals the following pattern: on highest educational attainment, while $82 \%$ of study participants from Masaka (rural site) had seven years or less of formal education, the figure for Entebbe (semi-urban site) was 63\% (a difference that was statistically significant); on socio-economic index (SEI), while the mean SEI of study participants from Masaka (rural site) was 13.9( $\mathrm{SD}=2.9)$ that for Entebbe (semi-urban site) was higher at 16.4 (a difference that was statistically significant). These results indicate that there are important socio-economic differences between the rural and urban study sites which may underlie the difference in incidence of MDD.

The risk of persistent of MDD over 12 months in this study was 24.6\%. Olley and colleagues (2006) [4] in South Africa in one of the few studies in this area reported a 6-months rate of persistence of MDD in HIV/AIDS of $44.8 \%$. The South African findings may have been confounded by the very high rate of loss to follow-up of 56\%. On the flip side, $75.4 \%$ of the MDD cases in this study 
Incidence and persistence of depression in HIV in Uganda

resolved by 12 months, the majority in less than 6 months (67\%). Similar results to those observed in this study have been reported in a general population cohort study in the Netherlands (the NEMESIS Study) where Spijker and colleagues (2002) reported that $76 \%$ of their study participants recovered from MDD by 12 months with the majority (63\%) of these recovering by 6 months [10]. Whiteford and colleagues (2013) in a systematic review on spontaneous remission of untreated MDD reported that 53\% of untreated cases of MDD remitted by 12 months while 32\% remitted by 6 months [37].

Apart from the natural life cycle of MDD, other possible reasons for the high remission rates of MDD observed in this study include that all participants diagnosed with MDD were offered health education about MDD (psychoeducation is known to be effective against MDD [38] and then referred to the nearest health facility that offered mental health care. Secondly, $43 \%$ of the study participants initiated antiretroviral therapy (ART) during the course of this study. Initiation of ART has previously been reported by Wagner and colleagues (2012) in the HIV care situation of Uganda to be associated with a significant reduction in depressive symptoms mainly mediated through improvement in physical health functioning [39].

In this study baseline depressive symptoms and marginally, study site (rural-urban difference), religion, a heavier weight, a family history of psychiatric disorder, higher negative life events and higher stress were associated with persistent MDD. At multivariate analysis study site, elevated baseline depressive scores and a heavier weight were independently associated with persistent MDD.

Of the observed predictor factors for persistence of MDD in HIV/AIDS in this study, only elevated depressive symptoms in early stage disease has previously been reported to be associated with persistent MDD in HIV/AIDS [11]. In this study, heavier weight was a predictor of persistence of MDD, an associated that has not been previously reported . Unlike the results from this study, the few adult prospective studies undertaken among non HIV populations reveal that MDD is more likely to elevate the risk of subsequent obesity than the converse [40-42]. From non HIV studies, stressful life 
Incidence and persistence of depression in HIV in Uganda

events have been reported to be a predictor of persistent MDD [43-44]. Just like in this study where a family history of psychiatric disorder was associated with persistent MDD, Lieb and colleagues (2002) in a German community study of adolescents and young adults reported that parental depression was associated with a more malignant course of depressive disorders in offspring [45]. The association between study site and persistence of MDD in this study probably points to the importance of ecological level factors in the persistence of MDD in HIV/AIDS in the African sociocultural context.

In conclusion, on the incidence of MDD, the rate obtained in this study was higher than that reported in two previous studies undertaken in Guadeloupe (in Central America) and French Guyana (in South America), countries with generalised HIV epidemics like that in Uganda, this probably points to differences in ecological risk factors for MDD. Secondly, the potentially modifiable factors of elevated baseline depressive scores and increased stress scores were independent predictors of incident MDD in this study. The later findings have at least two implications for depression management, firstly, that elevated depressive scores (even those that are below the threshold for MDD diagnoses) are a risk factor for future MDD episodes and should therefore be screened for and managed. Secondly, that stress (number and severity of negative life events in the previous 6 months) is risk factor for future MDD episodes and hence should be included in the risk assessment for MDD and where elevated managed.

In this study we reported rates of persistence of MDD that were similar to those reported in a nonHIV community sample in the Netherlands. The fact that about a quarter of the cases of MDD in this study were persistent at 6 months and up to 12 months and given the potentially negative clinical and psychosocial consequences of MDD reinforces the need to treat MDD in HIV/AIDS even in this African socio-cultural context. On the flip side however, given that three quarters of the cases of MDD remitted without instituting formal MDD treatment and given the severe challenges facing health systems in low and-middle income countries such as Uganda [46-47] calls for caution in the 
Incidence and persistence of depression in HIV in Uganda

recommendations we make for the management of MDD in such settings. In such settings there is need to institute stepped care collaborative management approach for MDD where low intensity therapies such as psychoeducation [48] and the Health Activity Programme [49] with demonstrated efficacy against MDD even when administered by lower level cadre such as general nurses and lay health workers can be offered to those with mild to moderate MDD and antidepressant medication and referral to mental health specialists offered to those with severe MDD. However, there is need for studies to develop and evaluate such stepped care collaborative models in the context of the health systems challenges facing HIV care services in sub-Saharan African settings such as those in Uganda. Lastly, there is need for studies to specifically delineate the factors underlying ecological risk for MDD in HIV/AIDS, and more broadly, to better understand ecological correlates of mental illness in the African situation.

As a limitations of this study, exclusion from enrolment of those with recent defaulted appointments may have introduced selection bias because MDD is a known risk factor for non-adherence with medical treatment [50]. Secondly, in designing this study, we assumed that the majority of MDD episodes run a cycle of 6 months based on previous work undertaken in the west among HIV negative general population samples [10]. Indeed in this study, the majority of MDD episodes (67\%) went into remission by 6 months although a small number (8.4\%) went into remission between 6 and 12 months. 
Incidence and persistence of depression in HIV in Uganda

\section{Acknowledgement}

This study was supported by a Senior Fellowship to Eugene Kinyanda from the European \& Developing Countries Clinical Trials Partnership (EDCTP) Project No. TA.2010.40200.011. We acknowledge the work and support provided by staff of the MRC/UVRI Mental Health Project. We would also like to acknowledge the support and corporation by the management and clients of The AIDS Support Organisation (TASO) Service Centres of Entebbe and Masaka. 
Incidence and persistence of depression in HIV in Uganda

\section{Reference}

1. Kinyanda E, Hoskins S, Nakku J, Nawaz S, Patel V. Prevalence and risk factors of major depressive disorder in HIV/AIDS as seen in semi-urban Entebbe district, Uganda. BMC Psychiatry. 2011;11:205.

2. Abas M, Ali GC, Nakimuli-Mpungu E, Chibanda D. Depression in people living with HIV in subSaharan Africa: time to act. Trop Med Int Health. 2014;19(12):1392-6.

3. Robertson K, Bayon C, Molina JM, et al. Screening for neurocognitive impairment, depression, and anxiety in HIV-infected patients in Western Europe and Canada. AIDS Care. 2014;26(12):155561.

4. Olley BO, Seedat S, Stein DJ. Persistence of psychiatric disorders in a cohort of HIV/AIDS patients in South Africa: a 6-month follow-up study. J Psychosom Res. 2006;61(4):479-84.

5. Atkinson JH, Heaton RK, Patterson TL, et al. Two-year prospective study of major depressive disorder in HIV-infected men. J Affect Disord. 2008;108(3):225-34.

6. Collins PY, Holman AR, Freeman MC, Patel V. What is the relevance of mental health to HIV/AIDS care and treatment programs in developing countries? A systematic review. AIDS. 2006; 20(12):1571-82.

7. Antelman G, Kaaya S, Wei R, et al. Depressive symptoms increase risk of HIV disease progression and mortality among women in Tanzania. J Acquir Immune Defic Syndr. 2007;44(4):470-7.

8. Mayston R, Kinyanda E, Chishinga N, Prince M, Patel V. Mental disorder and the outcome of HIV/AIDS in low-income and middle-income countries: a systematic review. AIDS. 2012;26 (Suppl 2):S117-35.

9. Nduna M, Jewkes RK, Dunkle KL, Shai NP, Colman I. Associations between depressive symptoms, sexual behaviour and relationship characteristics: a prospective cohort study of young women and men in the Eastern Cape, South Africa. J Int AIDS Soc. 2010;13:44. 
Incidence and persistence of depression in HIV in Uganda

10. Spiker J, de Graaf R, Bijl RV, Beekman ATF, Ormel J, Nolen WA. Duration of major depressive episodes in the general population: results from the Netherlands Mental Health Survey and Incidence Study (NEMESIS) Br J Psychiatry. 2002; 181:208-213.

11. Lyketsos CG, Hoover DR, Guccione M, et al. Depressive symptoms over the course of HIV infection before AIDS. Soc Psychiatry Psychiatr Epidemiol. 1996;31(3-4):212-9.

12. Rabkin JG, Goetz RR, Remien RH, Williams JB, Todak G, Gorman JM. Stability of mood despite HIV illness progression in a group of homosexual men. Am J Psychiatry. 1997;154(2):231-8.

13. Johnson JG, Rabkin JG, Lipsitz JD, Williams JB, Remien RH. Recurrent major depressive disorder among human immunodeficiency virus (HIV)-positive and HIV-negative intravenous drug users: findings of a 3-year longitudinal study. Compr Psychiatry. 1999;40(1):31-4.

14. Lyketsos CG, Hoover DR, Guccione M, et al. Changes in depressive symptoms as AIDS develops. The Multicenter AIDS Cohort Study. Am J Psychiatry. 1996;153(11):1430-7.

15. Tsao JC, Dobalian A, Moreau C, Dobalian K. Stability of anxiety and depression in a national sample of adults with human immunodeficiency virus. J Nerv Ment Dis. 2004;192(2):111-8.

16. Jin H, Atkinson JH, Duarte NA, et al. Risks and predictors of current suicidality in HIV-infected heroin users in treatment in Yunnan, China: a controlled study. J Acquir Immune Defic Syndr. 2013;62(3):311-6.

17. Elenga N, Georger-Sow MT, Messiaen T, et al. Incidence and predictive factors of depression among patients with HIV infection in Guadeloupe: 1988-2009. Int J STD AIDS. 2013;25(8):559-563.

18. Nacher M, Adriouch L, Godard Sebillotte C, et al. Predictive factors and incidence of anxiety and depression in a cohort of HIV-positive patients in French Guiana. AIDS Care. 2010;22(9):1086-92.

19. TASO Services and Programme.

http://www.tasouganda.org/index.php?option=com_content\&view=article\&id=72\&Itemid=218. 
Incidence and persistence of depression in HIV in Uganda

20. Monroe SM, Simons AD. Diathesis-stress theories in the context of life stress research: implications for the depressive disorders. Psychol Bull. 1991;110(3):406-25.

21. World Health Organization. WHO case definitions of HIV for surveillance and revised clinical staging and immunological classification of HIV-related disease in adults and children. http://www.who.int/hiv/pub/guidelines/HIVstaging150307.pdf. 2007.

22. Sacktor NC, Wong M, Nakasujja N, et al. The International HIV Dementia Scale: a new rapid screening test for HIV dementia. AIDS. 2005;19(13):1367-74.23.

23. Sheehan DV: Sheehan Disability Scale (SDS) In: Rush JA, Pincus HA, First MB, et al, eds. The Handbook of Psychiatric Measures. $1^{\text {st }}$ ed. Washington, DC: American Psychiatric Association; 2000:113-115.

24. Bernstein DP, Fink L. Childhood Trauma Questionnaire: A retrospective self-report manual. The Psychological Corporation, San Antonio, TX; 1998.

25. Dahlem NW, Zimet GD, Walker RR. The Multidimensional Scale of Perceived Social Support: a confirmation study. J Clin Psychol. 1991;47(6):756-61.

26. Wright K, Naar-King S, Lam P, Templin T, Frey M. Stigma scale revised: reliability and validity of a brief measure of stigma for HIV+ youth. J Adolesc Health. 2007;40(1):96-8.

27. Carver CS. You want to measure coping but your protocol's too long: consider the brief COPE. Int J Behav Med. 1997;4(1):92-100.

28. Connor KM, Davidson JR. Development of a new resilience scale: the Connor-Davidson Resilience Scale (CD-RISC). Depress Anxiety. 2003;18(2):76-82.

29. Kerkhof AJFM, Bernasco W, Bille-Brahe U, Platt S, Schmidtke A: A WHO/EURO Multicentre study on parasuicide. European Parasuicide study interview schedule. EPSIS I version 6.2 In: H Schiødt H, Aagaard B, eds. Department of Clinical and Health Psychology, University of Leiden, 1989. 
Incidence and persistence of depression in HIV in Uganda

30. Kinyanda E, Hjelmeland H, Musisi S. Negative life events associated with deliberate self-harm in an African population in Uganda. Crisis. 2005;26(1):4-11

31. Sheehan DV, Lecrubier Y, Sheehan KH, et al. The Mini-International Neuropsychiatric Interview (M.I.N.I.): the development and validation of a structured diagnostic psychiatric interview for DSMIV and ICD-10. J Clin Psychiatry. 1998;59(Suppl 20):22-33.

32. Radloff LS. The CES-D scale: A self-report depression scale for research in the general population. Applied Psychological Measurement, 1977;385-401.

33. Victoria CG, Huttly SR, Fuchs SC, Olinto MTA. The role of conceptual frameworks in epidemiological analysis: A hierarchical approach. Int. J. Epidemiol. 1997; 26 (1): 224-227.

34. Rayston P, Ambler G, Sauerbrei W. The use of fractional polynomials to model continuous risk variables in epidemiology. Int. J. Epidemiol. 1999;28: 964-974.

35. Lackner JB, Joseph JG, Ostrow DG, Eshleman S. The effects of social support on Hopkins Symptom Checklist-assessed depression and distress in a cohort of human immunodeficiency viruspositive and -negative gay men. A longitudinal study at six time points. J Nerv Ment Dis. 1993;181(10):632-8.

36. Kinyanda E, Woodburn P, Tugumisirize J, Kagugube J, Ndyanabangi S, Patel V. Poverty, life events and the risk for depression in Uganda. Soc Psychiatry Psychiatr Epidemiol. 2011;46(1):35-44.

37. Whiteford HA, Harris MG, McKeon G, Baxter A, Pennell C, Barendregt JJ, Wang J. Estimating remission from untreated major depression: a systematic review and meta-analysis. Psychol Med. 2013;43(8):1569-85.

38. Ali BS, Rahbar MH, Naeem S, Gul A, Mubeen S, Iqbal A. The effectiveness of counseling on anxiety and depression by minimally trained counselors: a randomized controlled trial. Am J Psychother. 2003;57(3):324-36. 
Incidence and persistence of depression in HIV in Uganda

39. Wagner GJ, Ghosh-Dastidar B, Garnett J, Kityo C, Mugyenyi P. Impact of HIV antiretroviral therapy on depression and mental health among clients with HIV in Uganda. Psychosom Med. 2012;74(9):883-90.

40. Hasler G, Pine DS, Kleinbaum DG, Gamma A, Luckenbaugh D, Ajdacic V, Eich D, Rössler W, Angst J. Depressive symptoms during childhood and adult obesity: the Zurich Cohort Study. Mol Psychiatry. 2005; 10(9):842-50.

41. Luppino FS, de Wit LM, Bouvy PF, Stijnen T, Cuijpers P, Penninx BW, Zitman FG. Overweight, obesity, and depression: a systematic review and meta-analysis of longitudinal studies. Arch Gen Psychiatry. 2010; 67(3):220-9.

42. Needham BL, Epel ES, Adler NE, Kiefe C. Trajectories of change in obesity and symptoms of depression: the CARDIA study. Am J Public Health. 2010; 100(6):1040-6.

43. Husain N, Parveen A, Husain M, et al. Prevalence and psychosocial correlates of perinatal depression: a cohort study from urban Pakistan. Arch Womens Ment Health. 2011; 14(5):395-403.

44. Horwitz SM, Briggs-Gowan MJ, Storfer-Isser A, Carter AS. Prevalence, correlates, and persistence of maternal depression. J Womens Health. 2007;16(5):678-91.

45. Lieb R, Isensee B, Höfler M, Pfister H, Wittchen HU. Parental major depression and the risk of depression and other mental disorders in offspring: a prospective-longitudinal community study. Arch Gen Psychiatry. 2002;59(4):365-74.

46. Wagner GJ, Ngo V, Glick P, Obuku EA, Musisi S, Akena D: INtegration of DEPression Treatment into HIV Care in Uganda (INDEPTH-Uganda): study protocol for a randomized controlled trial. Trials 2014;15:248.

47. Kigozi FN, Ssebunnya J: Integration of mental health into primary health care in Uganda: opportunities and challenges. Mental Health Family Medicine 2009; 6(1):37-42.

48. 
Incidence and persistence of depression in HIV in Uganda

49. Ali BS, Rahbar MH, Naeem S, Gul A, Mubeen S, Iqbal A: The effectiveness of counseling on anxiety and depression by minimally trained counselors: a randomized controlled trial. American Journal of Psychotherapy 2003; 57(3):324-36.

50. Chowdhary N, Anand A, Dimidjian S, Shinde S, Weobong B, Balaji M, Hollon SD, Rahman A, Wilson GT, Verdeli H, Araya R, King M, Jordans MJD, Fairburn C, Kirkwood B, Patel V. The Healthy Activity Program lay counsellor delivered treatment for severe depression in India: systematic development and randomised evaluation. The British Journal of Psychiatry 2016;208, $381-388$.

46. Gonzalez JS, Batchelder AW, Psaros C, Safren SA. Depression and HIV/AIDS treatment nonadherence: a review and meta-analysis. J. Acquir. Immune. Defic. Syndr. 2011;58(2),181-7. 